\title{
Computer Aided Optimization/Innovation of Passive Tracking Solar Concentration Fresnel Lens
}

\author{
Noel León, Humberto Aguayo, Hector García, and Alán Anaya \\ CIDYT - Center for Innovation in Design \& Technology, \\ Tecnologico de Monterrey, Mexico
}

\begin{abstract}
A passive solar tracker and concentrator device must be able to effectively concentrate solar radiation into a constant area throughout the day, without any mobile mechanism. The objective is to achieve $1000{ }^{\circ} \mathrm{C}$ on the receptor area with concentrated solar energy, and to accomplish this target a compound Fresnel lens has been designed. Utilizing multidisciplinary CAE software, an integrated multi-objective optimization process based on genetic algorithms has been developed and a novel design solution has been achieved. The process consists of integrating genetic algorithms using optimization software Dakota from Sandia National Laboratories with Autodesk Inventor for parametric variation of the lens 3D-CAD geometry, Lambda Research TracePro software for ray tracing simulation, and Microsoft Excel to manage data input and output. Several simulation scenarios were developed, such as a solar tracker and concentrator device for two-dimensional (linear) concentration and threedimensional (spot) concentration on equatorial and non-equatorial locations.
\end{abstract}

Keywords: Optimized Fresnel lens, passive solar tracking, genetic algorithms, solar concentrator, computer-aided innovation, solar thermal energy.

\section{Introduction}

Most solar energy research has focused on photovoltaic (PV) cells, which generate electricity with a molecular chain reaction. This reaction is triggered by a solar energy flux that occurs on a thin layer of silicon or germanium based compound. However, commercial PV cells have only yet achieved a15\% to $20 \%$ efficiency rate [1].

Two main issues about solar thermal energy must be overcome. First, solar energy must be concentrated for thermal applications, this due the low-density nature of such energy. Second, for most solar concentrators, solar rays must fall perpendicular to the concentrator at all times. Therefore, a solar tracking device must be used. A third factor could be the uncertainty of weather conditions, as not all locations in the world have regularly sunny days during most of year.

This work was done to explore the feasibility of using a Fresnel lens as a solar concentrator and passive solar tracking device. The main objective of this research work is to develop an automated optimization process for a solar tracking and concentrator device that will concentrate solar thermal energy into a thermal tank. Subsequently, the thermal battery (or tank) will be used as a power source for a Stirling engine to produce electricity. A Stirling engine is a machine that transforms 
thermal energy to mechanical energy using a work fluid. Several systems have been developed previously for such propose [2, 3]. Focusing solar rays into a thermal battery instead of focusing them directly to the Stirling engine gives mobility to the system, which is more practical for electric generation applications. Also, it opens the possibility to use thermal stored energy for transportation vehicles.

The developed automated optimization process uses a 3D-CAD modeler, raytracing simulation software and a commercial genetic algorithm (GA) package. To understand the simulation parameters and how it works, some basic background information of solar concentration and GA is presented.

\subsection{Solar Concentration and Tracking}

There are two types of solar concentration linear and spot, where linear or 2D concentration refers to an area concentrated to a line and 3D concentration refers to an area concentrated to a spot. 3D concentration achieves the highest practical concentration level.

Solar irradiance intensity changes depending on geographical location, season of the year, weather conditions and time of day. The Liu, B. \& Jordan, R. model [4] was used to describe solar irradiance of Monterrey, Mexico (with a latitude of $25^{\circ} 40^{\prime} \mathrm{N}$ ).

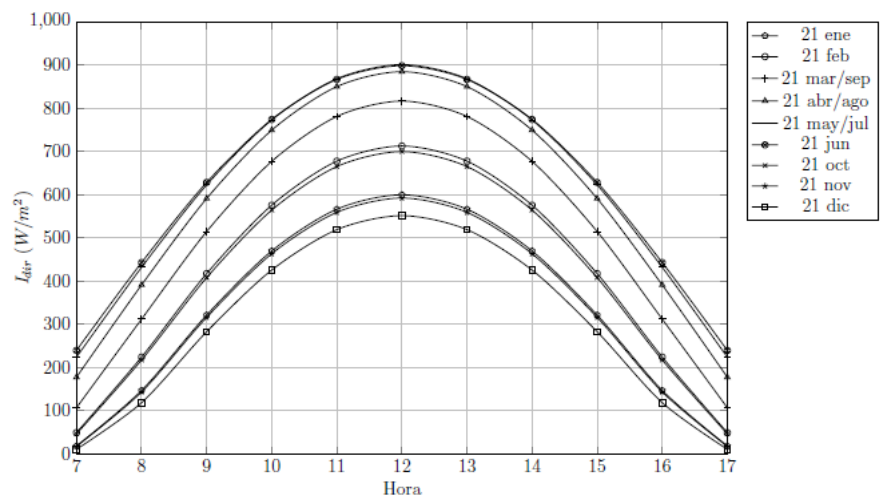

Fig. 1. Direct solar irradiance in $\mathrm{W} / \mathrm{m}^{2}$ (vertical axis) from 7:00 to 17:00 hours (horizontal axis) on different dates of the year in Monterrey, Mexico

As you can see, the highest irradiance intensities are between 9:00 hours and 15:00 hours. Thus the solar concentrator and passive tracking device is designed to work during that window of time.

To reach $1000^{\circ} \mathrm{C}$ on target with solar energy as the only power source is a matter of concentration levels and solar irradiance at a specific moment of the day. The following graph shows the concentration level versus temperature relation, using a basic thermodynamic model [5].

$$
T_{r, \max }=T_{s} \sqrt[4]{\frac{C}{c_{\max }}} .
$$

Where $T_{s}=5777 \mathrm{~K}$ is the temperature of the Sun's surface. 


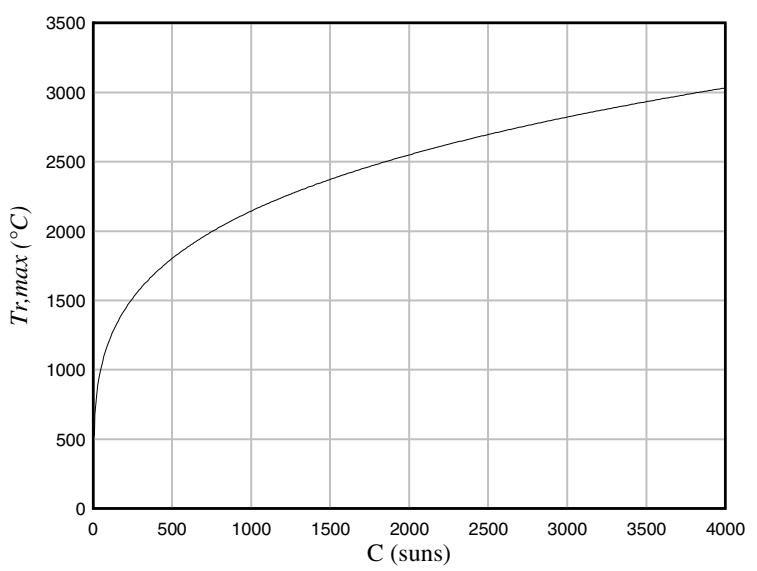

Fig. 2. Maximum thermodynamic temperature in ${ }^{\circ} \mathrm{C}$ (vertical axis) relative to the concentration level in suns (horizontal axis). No thermal losses were considered in this model [5]

According to the above model, to achieve $1000^{\circ} \mathrm{C}$ on target (focused area) a minimum concentration level of 100 suns is needed, which is essential for the design parameters of our solar concentration and passive tracking device.

To reach higher levels of solar concentration, typical solar collectors must decrease their acceptance half-angle. Therefore, it is necessary to follow the Sun's apparent movement respective to the Earth. There are different techniques employed depending of the level of concentration needed. Three main categories are listed below [6].

- Seasonal tracking

- Single axis tracking

- Double axis or ideal tracking.

This document presents a device with no movement, using Fresnel lenses to concentrate and to track the Sun's first axis of apparent trajectory. The second axis of trajectory (or seasonal axis) can be compensated by an inclination of the device towards the south (or to north at the southern hemisphere), where the inclination angle must be the same as the geographical latitude angle of the device's location (in our case Monterrey is roughly at $25^{\circ} 40^{\prime} \mathrm{N}$ ) [7].

\subsection{Fresnel Lens Exterior Shapes and Internal Prisms}

Dome-shaped (3D) or arched (2D) lenses can be designed when the prisms are chained along a semicircle centered at the focal point. A shaped lens offers advantages over flat lenses, such as increased mechanical stability with Fresnel grooves located inside for easy cleaning, and also reduces focal aberrations, but has the disadvantage of a more complex manufacturing process. Other more complex systems integrate closed loop control to change the refraction index of a liquid crystal material located between two layers of Fresnel lens grooves [12, 13]. To construct a 


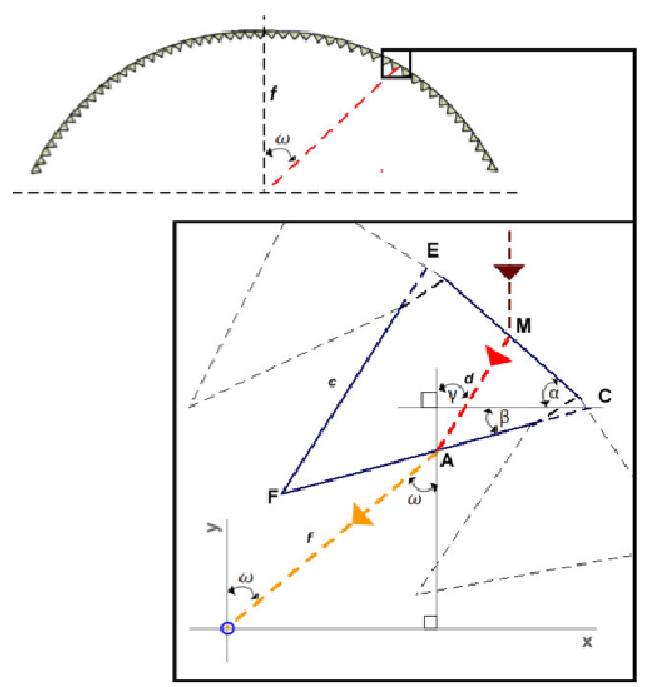

Fig. 3. Shaped Fresnel lens profile diagram [7]

shaped Fresnel lens, first the prism's angles and position must be calculated, as shown in the following diagram:

Based on the parameters depicted in Fig. 3, the following two equations can be set [4]:

$$
\begin{gathered}
\sin a=n \sin (a-\gamma) \\
n \sin (\beta-\gamma)=\sin (\beta+\omega)
\end{gathered}
$$

Where $n$ is the refraction index of the Fresnel Lens Concentrator (FLC) material. With $a=\omega$, eq. (2) can be solved for $\gamma$ via:

$$
\gamma=\omega-\sin ^{-1}\left(\frac{\sin \omega}{n}\right)
$$

Using sine theorems eq. (3) is solved for angle $\beta$ :

$$
\beta=\tan ^{-1}\left(\frac{n+1}{n-1} \tan \left(\frac{\omega-\gamma}{2}\right)\right)-\frac{\omega+\gamma}{2}
$$

With equations (4) and (5), for $\gamma$ and the prism inclination angle $\beta$ respectively, the design of the lens can be determined by the fixed focal length $f$ which in turn determines the prism's angle $a$ (as defined in Fig. 3). Using the above equations, a first shaped Fresnel lens model was drawn in Autodesk Inventor (CAD software) for its future optimization with GA.

Poly methyl methacrylate (PMMA) commonly known as acrylic, is the material chosen for the FLC, has a 1.49 refractive index at $0.587 \mu \mathrm{m}$ and transmits light up to $92 \%$ (in a $3 \mathrm{~mm}$ thick sheet) [7]. 


\subsection{An Ideal Final Result Approach}

There are several relevant contradictions that need to be overcome in order to find a best possible solution within the set range of boundaries. However, the most important contradiction is that the solar tracking device must follow the sun's apparent trajectory without any moving mechanism. This is not naturally possible due to the constantly changing angle of the solar rays in relation to the Sun's apparent trajectory throughout the day. Also, the refraction/reflection index is typically a constant material property; therefore, rays with different angles of incidence will be refracted or reflected to different locations. There are solar tracking methods that do not involve power consuming actuators, yet those systems have moving parts and for applications where a large area of absorption is needed (i.e. $30 \mathrm{~m}^{2}$ ), a system with moving parts becomes more expensive and less practical in residential locations.

From an ideal final result point of view we understand passive solar tracking as a system that does not involve moving parts, and therefore a "tracking but not tracking" solution is needed. A passive solar tracking and concentration device was idealized as a fixed (movement free) apparatus that concentrates solar energy with a constant focus location for high concentration ratios. A universal geometric shape of a Fresnel lens was developed, where the lens refracts all solar rays towards the same spot no matter the incidence angle of the solar rays. In order to find this universal geometric shape, the use of genetic algorithms was chosen.

\subsection{Genetics Algorithms}

Genetic algorithms (Gas) are search algorithms based on the mechanics of natural selection and natural genetics. In every generation, a new set of artificial individuals (represented as strings) is created using bits and pieces of the fittest of the previous generation. Gas efficiently exploit historical information to speculate on new search points with expected improved performance.

Genetic algorithms are implemented using computer simulations to reduce the research time of a best suited solution to a specific problem, in which members of a universe of possible solutions, called individuals, are represented by chromosomes. A simple GA that yields good results in many practical problems is composed of three operations: Selection, reproduction and mutation [8].

\section{Process Integration Using Dakota and Batch Scripts}

Dakota by Sandia National Laboratories is an open source program used to apply GA methods to the optimization problem of a FLC and passive solar tracking device. Dakota handles codification of FLC CAD parameters; however, an exterior process is needed as an objective function to evaluate the created individuals [9]. The exterior process is executed in sequence by a batch file (windows based command line file). The following diagram presents how the developed automated process works:

Once the process' cycle is completed, an evaluation (fitness) value is assigned to the individual (a set of parameters that conforms to the FLC CAD part). Dakota reads and stores the fitness value and then proceeds to the next iteration. 


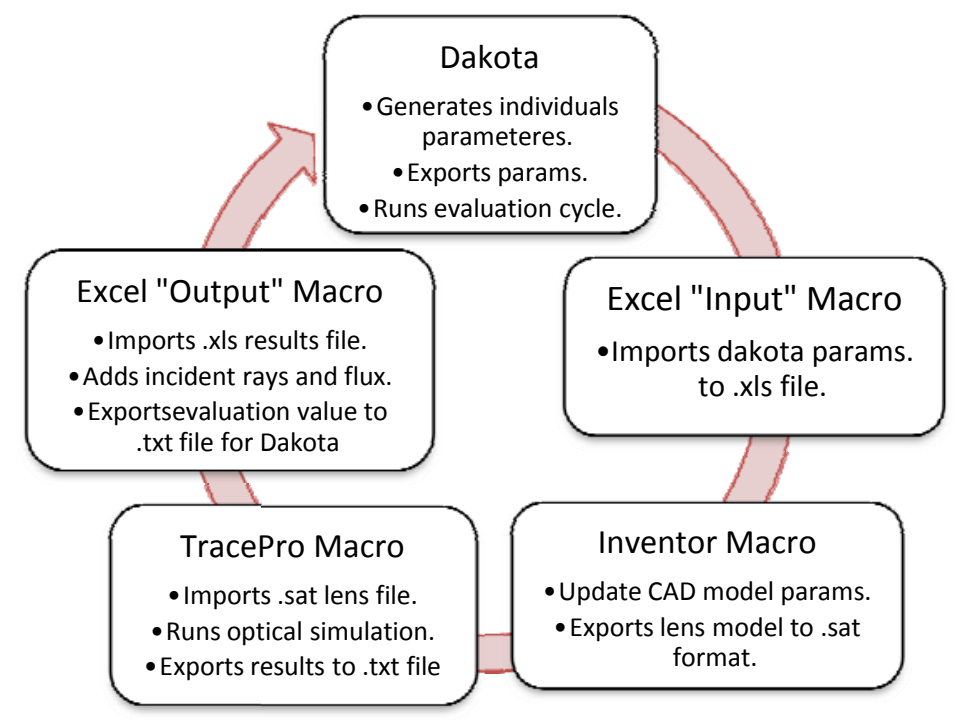

Fig. 4. Descriptive diagram of developed optimization process

Dakota has two commercial GAs optimization methods: Multi-Objective Genetic Algorithm (MOGA) and Single-Objective Genetic Algorithm (SOGA). Both were applied to different optimization scenarios described in the following section.

\subsection{FLC CAD Part Parameters}

Using section 1.4 equations, a CAD part was drawn and parameterized in Autodesk Inventor 2009 with general dimensions of $0.6 \mathrm{~m} \times 0.5 \mathrm{~m}$. The next image shows a section of the FLC profile and its parameters.

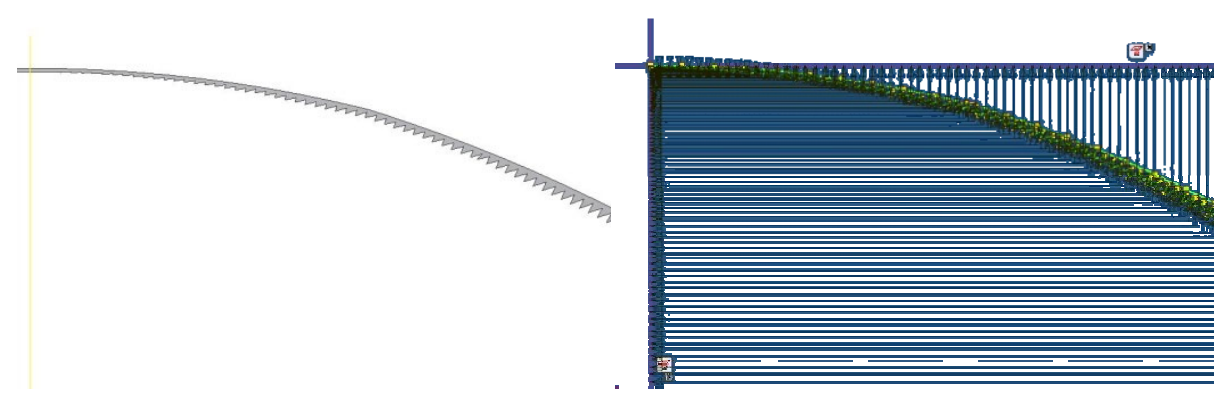

Fig. 5. FLC profile and its parameterization in respect to $X$ and $Y$ axis 
There are several ways to apply parameters to the FLC CAD part, and numerous ways were analyzed. A better optimization response was found by adding two dimensions to each prism, one respective to the " $X$ " (horizontal) axis and the other respective to the " $Y$ " (vertical) axis, with a total of 246 variables. The FLC profile was then mirrored in respect to the vertical axis. This dimensioning strategy gives the FLC profile fewer constraints, allowing its exterior curvature and internal prisms to change. Each parameter boundary is set up in Dakota's input file, thus SOGA or MOGA method only creates parameters within those sets of boundaries. When limits are too "loose" zero thickness parts are created, resulting in scrap iterations that do not provide any data to the optimization process. If limits are set too "tight" GA has a smaller universe of possible solutions, therefore the optimization process will not produce a significantly better individual compared with the initial model. A macro script was written in Visual Basic (VB) language to do the following actions:

1. Open Inventor template file with FLC part.

2. Update its parameters' values to the recently created individual by Dakota's MOGA or SOGA method.

3. Export a solid model of the updated FLC part in a neutral format (.sat).

4. Close Autodesk Inventor.

This macro is executed by a command line in a batch executable file [9].

\subsection{FLC Optics Simulation}

A template file with a ray-tracing simulation was done using Lambda Research TracePro v6.0 software. TracePro cannot simulate moving rays' sources. To simulate the Sun's apparent trajectory throughout the day, a scenario was build consisting of several rays' sources with properties of a solar angular profile and wavelength distribution from 0.3 to $0.6 \mu \mathrm{m}$ (visible light range) [10,11]. Rays' sources are created in a $15^{\circ}$ angle increment starting at $45^{\circ}$ with respect to the horizontal plane and ending at $135^{\circ}$, where each rays' source created 230 rays. Also, a specific irradiance value is set to each rays' source according to model in Figure 1. Table 1 shows the irradiance values on an average sunny day in Monterrey, Mexico, and these values were used for the intensity of the rays' sources in the TracePro simulation.

The next image shows the location and orientation of rays' sources in TracePro simulation template file:

Table 1. Irradiance values for solar rays sources during summer time in Monterrey, Mexico

\begin{tabular}{r|cc}
\hline Rays' source & Hour represented & Irradiance value $\left(\mathrm{W} / \mathrm{m}^{2}\right)$ \\
\hline $45^{\circ}$ & $9: 00$ & 630 \\
$60^{\circ}$ & $10: 00$ & 780 \\
$75^{\circ}$ & $11: 00$ & 870 \\
$90^{\circ}$ & $12: 00$ & 900 \\
$105^{\circ}$ & $13: 00$ & 870 \\
$120^{\circ}$ & $14: 00$ & 780 \\
$135^{\circ}$ & $15: 00$ & 630 \\
\hline
\end{tabular}



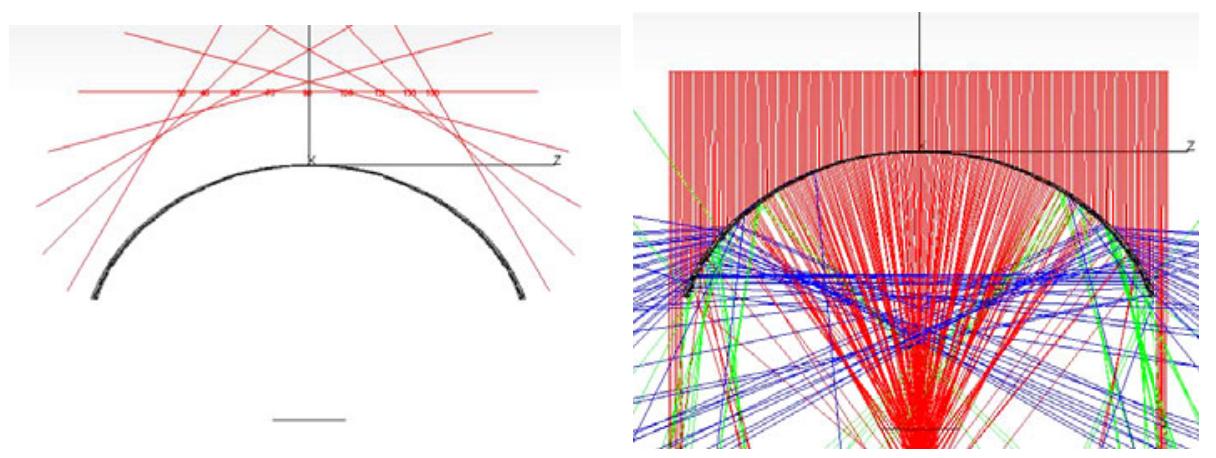

Fig. 6. Front view of rays' sources planes \& ray tracing simulation

The FLC solid model is inserted in the template file and moved to a location between the solar rays' sources and the receptor surface. The rays are generated perpendicular to its source plane towards the FLC model. The rays that fall into the FLC are refracted and concentrated on the receptor plane, which maintains a constant size and location. TracePro analyzes the information about the incident rays flux at the receptor surface. After the simulation is complete, TracePro generates a table with a list of all incident rays at the receptor surface, their original ray source, power fraction and the total power concentrated at the receptor surface.

To complete these actions without any user involvement a macro was programmed (macros in TracePro must be written in LISP programming language).

The macro script actions are listed below:

1. Open simulation template file.

2. Insert FLC solid model.

3. Apply material properties to FLC model.

4. Generate ray sources.

5. Run simulation.

6. Export incident rays data to an ASCCI format file.

7. Close TracePro.

Similar to Autodesk Inventor macro, TracePro macro is executed with a command line in the simulation cycle batch file. An Excel macro is used to extract key information of the total incident rays and the total incident flux from the ASCCI file. For the optimization process using SOGA method, the total incident rays were taken as the result of the objective functions. For MOGA method, the total incident flux was added as a second objective function. These values were assigned to the present individual at the end of each optimization process cycle. Excel macro is executed with a command line from the batch file.

\subsection{Batch Script}

Dakota input file for this optimization process has an interface section that points to a Windows 7 OS batch script file. This file contains command lines for execution of the simulation cycle used by Dakota to evaluate the present individual. Cycle batch file ("cycle.bat") holds the next lines: 
/ Cycle to evaluate created FLC by GA methods

cd $\mathrm{C}: \backslash$ Tesis $\backslash$ Batchscripts $\backslash$

call ImportParamtoExcel.bat

call OpenInventor.bat

call RunSimTracePro.bat

call Results.bat

call Delete.bat

Each line calls for another batch file to run each step of the simulation cycle. ImportParamtoExcel.bat executes an Excel macro that changes the format of Dakota's generated ASCCI file to a format that Autodesk Inventor macro understands. Results.bat executes an Excel macro to extract the objective functions values and export them to an ASCCI file that Dakota understands. Delete.bat is a subroutine that deletes temporary files created by the optimization process. It is done to avoid same file name issues. Other sub-routines are already detailed above.

\section{Results}

The FLC model constructed using equations described in section 2.2 was used as a template file and as a base model to be compared with the GA optimized model. Both models were tested using the developed TracePro macro (detailed in section 2.4).

An optimization process was performed using Dakota's SOGA method. The objective function was set to maximize the incident rays at the receptor plane. After more than 1,660 cycles, the FLC with the next fitness values were created:

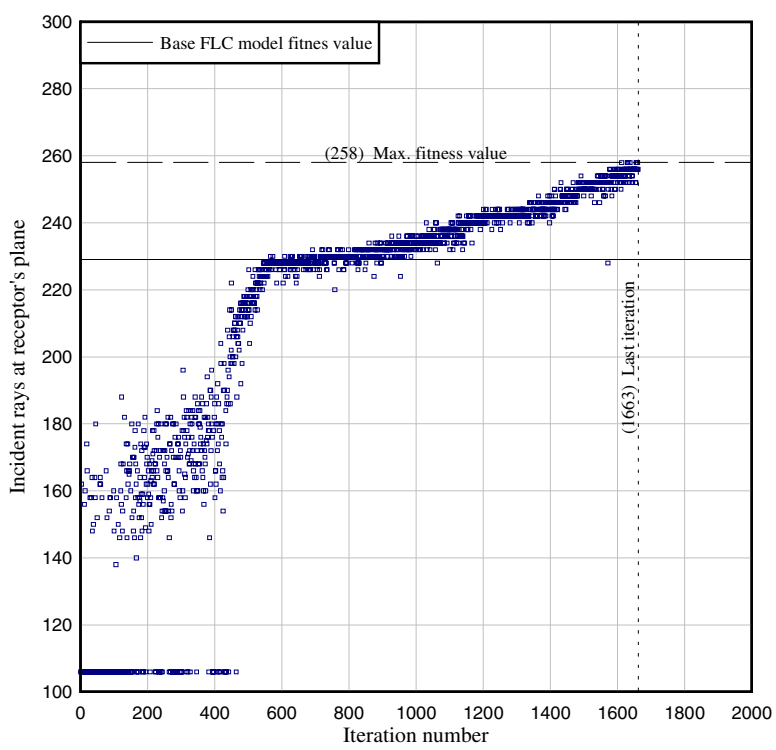

Fig. 7. FLC fitness value progress using SOGA method. Objective function: Maximize incident rays at the receptor's plane 
The above chart represents the fitness value of each FLC created by the optimization process. SOGA parameters must be setup in Dakota's input file; a copy of such parameters is presented next:

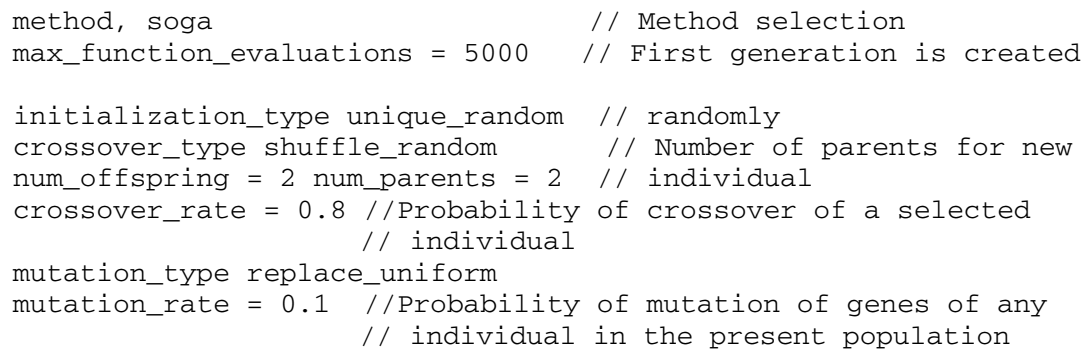

An improvement of FLC design can be seen after 500 cycles. After 1663 cycles, SOGA termination parameters concluded that no further significant improvement can be found and optimization process was concluded. The best individual was ranked with a fitness value of 258 incident rays at the receptor's plane. The same simulation macro ranked the base FLC model with a fitness value of 229. Comparing these two values GA FLC is about $12 \%$ more efficient that the base FLC model.

Incident rays do not necessary represent the amount of power being concentrated at the receptors plane since all rays, after been refracted by the FLC, loose a different amount of power depending on its incident angle and FLC material properties (refraction index, absorption, etc). To take account for the concentrated power at the receptor's plane, Dakota's MOGA method (Multiple Objective Genetic Algorithm) was used. Maximizing incident flux and rays at the receptor plane were set as the second object function for the next optimization process. Each rays source generated 230 rays with the equivalent of 1 watt of power per ray in order to count the flux concentration. After more than 1200 cycles, the optimization process had the next fitness improvement with respect to the base FLC:

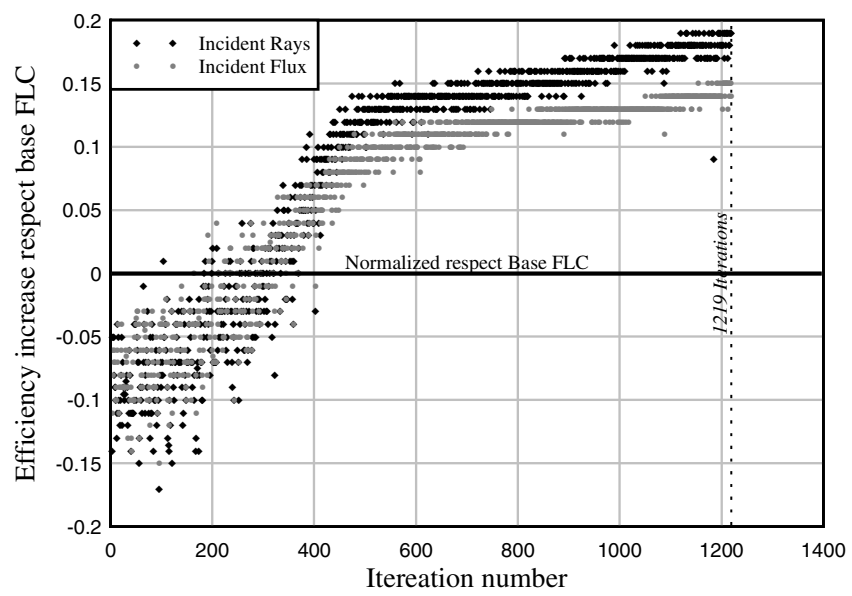

Fig. 8. GA FLC fitness values progress using MOGA method. Each dot represents a FLC model (individual) created by the GA optimization process. 
MOGA's method parameters were set up similar to SOGA's. Using two objective functions in the optimization process resulted in a FLC optimized model with an increment of the Incident Rays' fitness value to $20 \%$ and the Incident Flux to $16 \%$, compared to the base FLC model. A series of images of the fittest individual found in the optimization process is shown below.
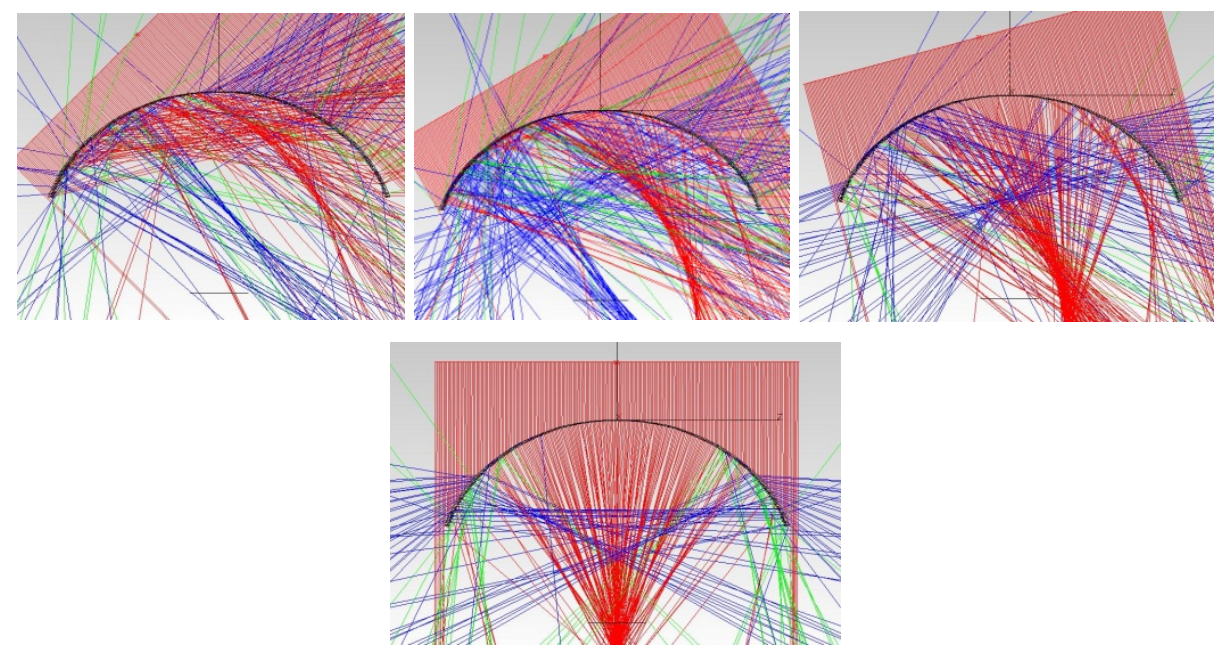

Fig. 9. Ray-tracing simulation with rays sources at $45^{\circ}, 60^{\circ}, 75^{\circ}$ and $90^{\circ}$ respectively. Results for rays' sources at $105^{\circ}, 120^{\circ}$ and $135^{\circ}$ can be obtained by mirroring above simulation results by its vertical axis. Red rays have a $66 \%$ to $100 \%$ of the original ray (prior refraction) flux; blue rays have a $33 \%$ to $66 \%$ of the original ray flux.

TracePro ray tracing simulation shows the development of a second focus (marked with blue rays) that does not exist in the base FLC model, refracting more rays towards the receptor's plane. The location of the principal focus (marked with red rays) moves due a change of the incident angle at which rays reach the exterior surface of the FLC, reducing significantly its overall efficiency. The following table

Table 2. Ray and flux concentrations overall efficiency in TracePro simulation scenario

\begin{tabular}{c|ccc}
\hline $\begin{array}{c}\text { Rays Source } \\
\text { Inclination } \\
\text { Angle }\end{array}$ & \multicolumn{3}{|c}{$\begin{array}{c}\text { Percentage of incident flux respect } \\
\text { refracted flux by the FLC }\end{array}$} \\
\cline { 2 - 4 } & $\begin{array}{c}\text { FLC Base } \\
\text { Model }\end{array}$ & $\begin{array}{c}\text { GA FLC } \\
\text { Model }\end{array}$ & $\begin{array}{c}\text { Without } \\
\text { any device }\end{array}$ \\
\hline $45^{\circ}$ & $2.33 \%$ & $5.35 \%$ & $6 \%$ \\
$60^{\circ}$ & $2.84 \%$ & $24.29 \%$ & $7 \%$ \\
$75^{\circ}$ & $3.48 \%$ & $4.19 \%$ & $8 \%$ \\
$90^{\circ}$ & $67.36 \%$ & $62.83 \%$ & $8 \%$ \\
$105^{\circ}$ & $3.48 \%$ & $4.19 \%$ & $8 \%$ \\
$120^{\circ}$ & $2.84 \%$ & $24.29 \%$ & $7 \%$ \\
$135^{\circ}$ & $2.33 \%$ & $5.35 \%$ & $6 \%$ \\
\hline Average & $\mathbf{1 2 . 0 9 \%}$ & $\mathbf{1 8 . 6 4 \%}$ & $\mathbf{6 . 9 6 \%}$ \\
\hline
\end{tabular}


compares the efficiency of base FLC model, the GA optimized FLC model, and the case of not using any device to concentrate rays into the receptor's plane.

The difference between GA FLC and conventional FLC may seem insignificant due the movement of focus and the fixed receptor's plane. However, the receptor plane only took an average of $12.1 \%$ of the total refracted power using the calculated base FLC and $18.6 \%$ with GA FLC, which is about 2.6 times more than not using any device. Another approach is to add more receptor planes at key locations, increasing the overall efficiency by reflecting concentrated rays toward a new receptor without shadowing each other. The next image shows the location of multiple receptors:

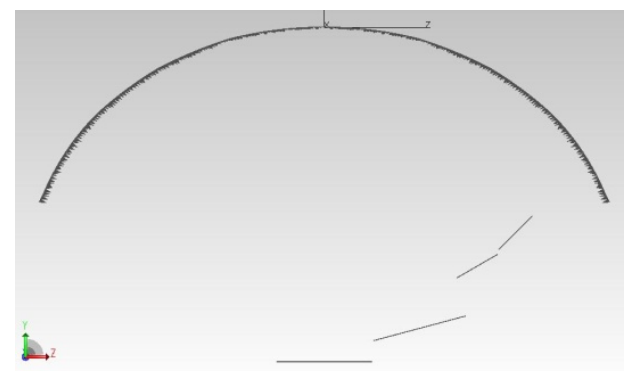

Fig. 10. Multiple receptors positioned to reflect concentrated rays toward a single fixed spot. Only half of the receptors are being used for the sake of the simulation; the other half can be mirrored by the FLC vertical axis (y axis).

Using the above configuration, most of the rays and flux refracted by the FLC are being concentrated into the receptors. The overall efficiency is shown in Table 3 . Receptors can be Fresnel mirrors, which are capable of reflecting incident rays and flux into a fixed receptor.

Table 3. Overall efficiency using multiple receptors for GA optimized FLC

\begin{tabular}{|c|c|c|c|c|c|c|}
\hline \multirow{2}{*}{$\begin{array}{c}\text { Source } \\
\text { Inclination } \\
\text { (Degrees) }\end{array}$} & \multirow[b]{2}{*}{$\begin{array}{l}\text { Time } \\
\text { (Hours) }\end{array}$} & \multicolumn{5}{|c|}{ Incident Flux percentage relative to GA FLC refracted flux } \\
\hline & & $\begin{array}{c}\text { Receptor } \\
1\end{array}$ & $\begin{array}{c}\text { Receptor } \\
2\end{array}$ & $\begin{array}{c}\text { Receptor } \\
3\end{array}$ & $\begin{array}{c}\text { Receptor } \\
4\end{array}$ & $\begin{array}{c}\text { Total } \\
\text { Efficiency }\end{array}$ \\
\hline 45 & 9:00 & $5.35 \%$ & $3.15 \%$ & $0.99 \%$ & $15.06 \%$ & $24.55 \%$ \\
\hline 60 & 10:00 & $4.29 \%$ & $3.69 \%$ & $26.46 \%$ & $9.60 \%$ & $44.04 \%$ \\
\hline 75 & 11:00 & $5.62 \%$ & $62.14 \%$ & $3.49 \%$ & $3.21 \%$ & $74.46 \%$ \\
\hline 90 & 12:00 & $62.83 \%$ & $7.09 \%$ & $2.17 \%$ & $1.75 \%$ & $73.84 \%$ \\
\hline 105 & 13:00 & $5.62 \%$ & $62.14 \%$ & $3.49 \%$ & $3.21 \%$ & $74.46 \%$ \\
\hline 120 & $14: 00$ & $4.29 \%$ & $3.69 \%$ & $26.46 \%$ & $9.60 \%$ & $44.04 \%$ \\
\hline 135 & 15:00 & $5.35 \%$ & $3.15 \%$ & $0.99 \%$ & $15.06 \%$ & $24.55 \%$ \\
\hline & & & & & Average: & $51.5 \%$ \\
\hline
\end{tabular}

\section{Conclusions and Future Work}

A final ideal result of a passive tracking device has not yet been reached. As can be seen in the results section, concentrated rays spot focus moves in a three dimensional trajectory depending on the hour of the day (due to Earth's principal rotational 
movement) and season of the year (due the tilt of the Earth's axis). Nevertheless, applying GA to a shaped Fresnel lens created a more efficient device to concentrate and track solar energy. The strategy used of dimensioning and constraining the FLC profile gave enough freedom to the profile that it allowed the GA methods to create better fitted individuals (FLCs).

A double layer FLC was developed to accomplish spot concentration with a single curvature device. For future work, additional stages of concentration, either a third layer of Fresnel lens or a mirror to the system, can be applied. Using a first concentration stage of 40 suns and a second of 2.5 suns will give a total of 100 suns, enough to achieve $1000^{\circ} \mathrm{C}$ on the final receptor with a minimal 4 to 8 percent of extra energy loss due the use of a second reflection or refraction respectively (estimated by PMMA refraction index and a typical mirror film reflection index) [14].

The FLC's main objective is to concentrate solar rays into a fixed thermal tank for thermodynamic purposes. Concentrated rays must enter the tank from below; therefore, an upwards reflection of concentrated rays must be done after the refraction of solar rays by the FLC. Independent Fresnel mirrors can be set on the focus trajectory with specific orientation to reflect FLC concentrated rays toward the fixed tank. Such mirrors' design and position must be calculated and optimized to minimize energy losses. A more elaborated ray tracing simulation must be accomplished to help the GA to generate FLC models that refract rays closer to the receptors.

\section{Acknowledgments}

Lambda Research for supporting research aimed to help human development and for the licensed copy of TracePro. Autodesk for donating free software licenses to ITESM students. To the Research Chair of Design and Innovation in Engineering (CAT101) for directly supporting this work.

\section{References}

1. Green, M., Emery, K., Hishikawa, Y., Warta, W.: Progress in Photovoltaics: Research and Applications Solar. Cell Efficiency Tables 17(5), 320-326 (2009)

2. Mills, D.: Advances in solar thermal electricity technology. Solar Energy Journal 76(1-3), 19-31 (2004)

3. Yogi-Goswami, D.: Solar Thermal Power Technology: Present Status and Ideas for the Future. Energy Sources, Part A: Recovery, Utilization, and Environmental Effects 20(2), 137-145 (1998)

4. Liu, B., Jordan, R.: The interrelationship and characteristic distribution of direct, diffuse and total solar radiation. Solar Energy Journal (1960)

5. Leutz, R., Suzuki, A.: Non-imaging Fresnel Lenses: Design and Performance of Solar Concentrators. Springer, Heidelberg (2001)

6. Chemisana, D.: Diseño y caracterización de un concentrador térmico - fotovoltaico cuasiestacionario para integración arquitectónica. Ph.D. Thesis. Universitat Lleida (2009)

7. Delgadillo, G.: Passive Tracking Enhanced Solar Concentrator Device Performed by Fresnel lens. Unpublished thesis material, ITESM University, Monterrey (2009) 
8. Valenzuela, M.: Algoritmo Genético Simple, Sistemas Conexionistas y Evolutivos: unpublished class material, ITESM University, Monterrey (2007)

9. Aguayo, H.: Optimization and Innovation in Engineering Design Consulting on Product Development. Ph.D. Thesis. ITESM University, Monterrey (2009)

10. Gordon, J.: Solar Energy: The State of the Art. ISES. James \& James Ltd (2001)

11. Thirugnanasambandam, M., Iniyan, S., Goic, R.: A Review of Solar Thermal Technologies. Elsevier, Amsterdam (2009)

12. Method for Light Ray Steering. Patent: US2007/0157924. SolBeam Inc., Assignee (2007)

13. Method and System for Light Ray Concentration. Patent: US2009/0250094. SolBeam Inc., Assignee (2009)

14. Bader, R., Haueter, P., Pedretti, A., Steinfeld, A.: Optical Design of a Novel. Journal of Solar Energy Engineering 131 (2009) 